\title{
Interaction lumière-température et influence de la photopériode sur le taux de croissance de Chlorella sorokiniana Shih. et Kraus.
}

\author{
M. Belkoural \\ A. Dauta ${ }^{2}$
}

Mots clés : Chlorella sorokiniana, taux de croissance, lumière, température, photopériode.

L'interaction de la lumière et de la température ainsi que l'effet de la photopériode sur la croissance de Chlorella sorokiniana sont étudiés en milieu fini et en conditions nutritives non limitantes. Le taux maximal de croissance $\left(2,7 \mathrm{j}^{-1}\right)$ est enregistré à la température de $35^{\circ} \mathrm{C}$, sous une intensité de $300{ }_{\mu} \mathrm{E} \cdot \mathrm{m}^{-2} \mathrm{~s}^{-1}$ et en éclairement continu. L'ensemble des données est repris sous la forme d'un modèle mathématique qui permet d'estimer le taux de croissance de la culture en fonction des conditions environnementales.

The influence of daylength, light intensity and temperature on the growth rate of Chlorella sorokiniana Shih. et kraus.

Keywords : Chlorella sorokiniana, growth rate, light, temperature, daylength.

The light and temperature interactions and the effect of daylength on the growth rate of Chlorella sorokiniana were studied in batches and in non-nutrient-limited conditions. The maximum growth rate $\left(2.7 \mathrm{~d}^{-1}\right)$ was recorded at a temperature of $35^{\circ} \mathrm{C}$, light intensity of $300 \mu \mathrm{E} \cdot \mathrm{m}^{-2} \cdot \mathrm{s}^{-1}$ and continuous light. The results were adjusted by a mathematical model which can be used to estimate the growth rate of a culture in relation to environmental conditions.

\section{Introduction}

La croissance d'une culture est contrôlée par un très grand nombre de facteurs dont les plus importants sont la lumière (intensité et photopériode), le $\mathrm{pH}$, les nutriments, les concentrations en $\mathrm{CO}_{2}$ et $\mathrm{O}_{2}$ et l'état physiologique (Markl 1980). L'évolution complexe du statut d'une population d'algues dans un système de culture est définie par une fonction logistique résultant de paramètres ayant des effets positifs ou négatifs au cours du temps. Ceci a des implications dans l'optimisation du rendement des cultures en masse utilisées dans la biotechnologie algale.

L'utilisation des microalgues à des fins appliquées (aquaculture, industries chimiques et pharmaceutiques) s'est nettement développée au cours de la dernière décennie (Soeder 1980, De Pauw et al. 1984,

1. Laboratoire du Physiologie végétale, Faculté des Sciences Semlalia, Avenue du Prince Moulay Abdellah, B.P. S 15, Marrakech, Maroc.

2. Laboratoire d'Hydrobiologie, URA 695 CNRS, Université Paul Sabatier, 118, route de Narbonne, 31062 Toulouse Cedex, France.
Becker 1986, Borowitzka 1986, Cohen 1986). Si ces microorganismes représentent en effet une source non négligeable de protéines (40 à $50 \%$ du poids sec), ils produisent par ailleurs des substances à haute valeur ajoutée (pigments, acides gras, vitamines, acides aminés...) qui intéressent les industries chimiques et pharmaceutiques. La valorisation des microalgues demeure cependant tributaire de la parfaite connaissance de leur composition biochimique et de leurs caractéristiques écophysiologiques spécifiques. C'est dans ce contexte que se situe le travail présenté dont l'objectif était de déterminer les conditions d'éclairement et de température optimales pour la culture de Chlorella sorokiniana, espèce potentiellement intéressante sur le plan industriel (Soong 1980, Richmond 1986).

De nombreux travaux consacrés à l'étude des interactions entre la lumière, la température et les nutriments sur la croissance de diverses espèces d'algues ont montré que le taux de croissance $(\mu)$ instantané d'une population pouvait être décrit comme une fonction d'un taux maximal spécifique ( $\mu$ max) affecté de facteurs de réduction liés aux variables 
environnementales et aux éléments nutritifs (Morgan \& Kalff 1989, Guerri et al. 1981, Dauta et al. 1990) :

$\mu=\mu \max * \operatorname{Red} \mathrm{T} * \operatorname{Red} \mathrm{I} * \operatorname{Red} \mathrm{N} * \operatorname{Red} \mathrm{P}(1)$ $\langle$ - terme 1 - > <- terme 2 - >

où Red T, Red I, Red $\mathrm{N}$ et Red $\mathrm{P}$ représentent la réduction du taux de croissance $\mu$ max par des conditions non optimales.

Dans des conditions nutritives non limitantes, l'action du terme 2 s'annule et la croissance devient une simple fonction de la lumière et de la température (Dauta 1982).

\section{Matériel et méthodes}

Chlorella sorokiniana ${ }^{1}$ a été isolèe des bassins de lagunage de la ville de Marrakech (Maroc). La souche est cultivée en milieu fini, sur un milieu entièrement minéral (Dauta 1982). Les cultures sont fortement bullées $\left(0,5\right.$ I d'air par litre de culture. $\left.\mathrm{mn}^{-1}\right)$ avec de l'air comprimé filtré (filtre Wathman GF/C de $1 \mu \mathrm{m}$ ) insufflé à la base des flacons. Les expériences sont réalisées dans des enceintes thermorégulées, sous une photopériode de 15/9 (15 heures de lumière pour 9 heures d'obscurité). L'éclairement est dispensé par des lampes PhytoClaude de 400 watts placées à la verticale des cultures. La mesure des intensités lumineuses incidentes a été réalisée à l'aide d'un quantamètre Li-Cor 185A ; la mesure, effectuée à la place du flacon, est corrigée pour tenir compte de la réflexion de la lumière sur les parois. Pour les expérimentations, les flacons sont obturés par du film transparent. La souche mère utilisée est maintenue en phase exponentielle de croissance par des repiquages fréquents ( 2 à 3 jours) dans des conditions d'éclairement et de température suboptimales.

Avant l'expérience destinée à la mesure du taux de croissance $(I, T)$, la culture est placée pendant 24 heures à l'obscurité (synchronisation et nonpréadaptation à la lumière). Au début de l'expérience, la culture est largement diluée dans un milieu neuf afin d'éviter tout effet d'autoombrage, puis répartie dans une série de flacons (erlenmeyers de $300 \mathrm{ml})$. Pour chaque température $(10,15,20,25$, $30,35^{\circ} \mathrm{C}$ ), les flacons sont exposés à des intensités lumineuses comprises entre 10 et $600{ }_{\mu} \mathrm{E} \cdot \mathrm{m}^{-2} \cdot \mathrm{s}^{-1}$.

1. Détermination réalisée par le Professeur E. Kessler, de l'Institut für Botanik und Pharmazeutische Biologie, Erlangen, Allemagne, que nous remercions.
Une expérience complémentaire a été effectuée afin d'étudier l'influence du nombre d'heures d'éclairement sur le taux de croissance : les flacons sont placés à $30^{\circ} \mathrm{C}$, sous une intensité lumineuse de $300 \mu \mathrm{E} . \mathrm{m}^{-2} \cdot \mathrm{s}^{-1}$ et à différentes photopériodes $(3 / 21$, $6 / 18, \ldots, 24 / 0)$.

Le taux de croissance est déterminé à partir de la densité optique des cultures (mesurée à $750 \mathrm{~nm}$ avec une cuve de $5 \mathrm{~cm}$ ), avec une première mesure au début du cycle lumineux $\left(A_{0}\right)$ et une deuxième après 24 heures d'incubation $\left(A_{1}\right)$. Le taux de croissance est calculé par la formule :

$$
\mu=\ln \left(\mathrm{A}_{1} / \mathrm{A}_{0}\right) \text {, }
$$

pour un intervalle de temps de 1 jour

\section{Résultats}

La figure 1 représente les modalités de variation du taux de croissance en fonction de l'énergie lumineuse reçue, pour chaque température. Le modèle mathématique (eq.3) proposé par Peeters \& Eilers (1978) permet un bon ajustement aux points expérimentaux, avec l'augmentation du taux de croissance en fonction de l'intensité lumineuse d'une part, et la prise en compte de l'effet inhibiteur des fortes intensités lumineuses d'autre part.

$$
\begin{gathered}
\mu(\mathrm{T}, 15 / 9)= \\
\mu \max * 2 *(1+\beta) * \mathrm{I}^{\prime} /\left(\mathrm{I}^{\prime 2}+2 * \mathrm{I}^{\prime} * \beta+1\right) \\
\text { avec I' }=\mathrm{I} / \mathrm{Iopt}
\end{gathered}
$$

Le tableau 1 donne la variation des valeurs des paramètres de cette équation. Pour chacune des températures testées, il est possible de déterminer un taux de croissance optimal $\left(\mu \max _{\mathrm{T}}\right)$ et une intensité lumineuse optimale associée $\left(\operatorname{Iopt}_{\mathrm{T}}\right)$. Les figures 2 et 3 représentent l'ajustement par le modèle (eq.4) de Lehman et al. (1975) aux variations de $\mu \max _{\mathrm{T}}$ et $\operatorname{lopt}_{\mathrm{T}}$ en fonction de la température. Le tableau 2 donne les valeurs des paramètres pour les deux sousmodèles.

$$
\begin{aligned}
\mu \max _{\mathrm{T}} & =\mu \max ^{*} \mathrm{e}\left(-2.3^{*}(\mathrm{~T}-\mathrm{Topt})^{2} / \mathrm{B}^{2}\right) \\
\text { avec B } & =\text { Tsup - Topt si T }>\text { Topt } \\
& =\text { Tinf - Topt si T }<\text { Topt, } \\
\mu \max & =\text { taux spécifique maximal de croissance, } \\
& \text { Topt est la température optimale, }
\end{aligned}
$$

Tinf et Tsup étant respectivement les températures inféricure et supéricures pour lesquelles $\mu \max _{\mathrm{T}}$ $=0.1 * \mu \max$ 
L'analyse des figures 2 et 3 met en évidence l'accroissement particulièrement important du taux de croissance et de la lumière optimale associée avec la température ; la valeur asymptotique correspondant à $35^{\circ} \mathrm{C}$. Le tableau 3 résume les principaux paramètres associés à la croissance de Chlorella
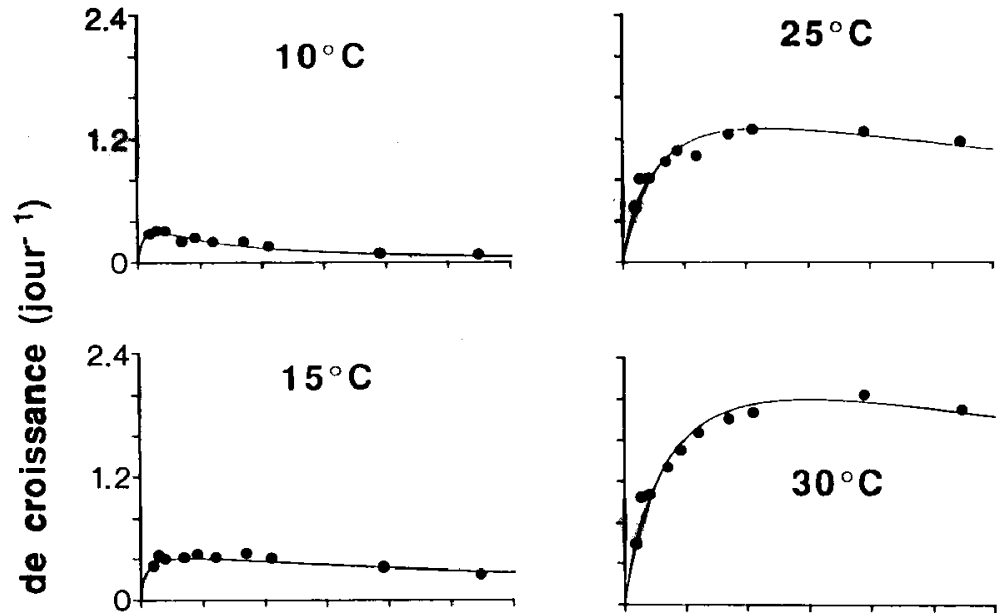

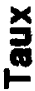

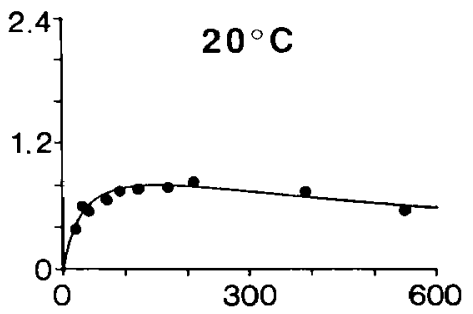

sorokiniana. La valeur du $\mathrm{Q}_{10}$ a été calculée à partir des résultats obtenus sur l'intervalle $20-30^{\circ} \mathrm{C}$. Le temps de génération (TG) a été déduit de la valeur de $\mu \max _{\mathrm{T}}$ (avec $\mathrm{T}=35^{\circ} \mathrm{C}$ ) par la formule : $\mathrm{TG}=\mu^{-1 *} \operatorname{Ln} 2$ (Reynolds 1984).
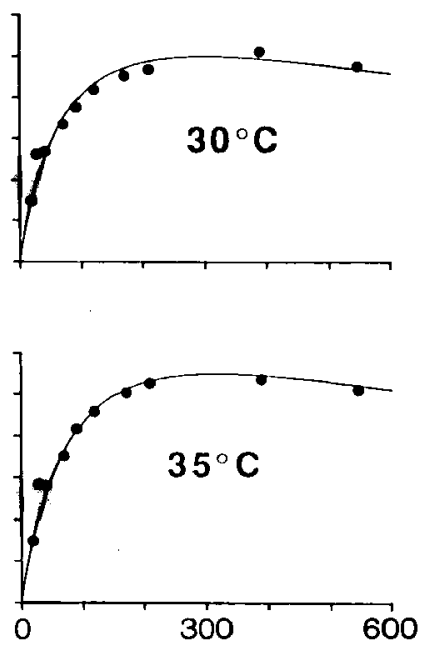

Intensité lumineuse ( $\mu$ E.m- . $^{-1}$ )

Fig. 1. Chlorello sorokiniana : Interaction de la lumière et de la température sur le taux de croissance (ajustement au modele de Peeters \& Eiters 1978).

Fig. 1. Chiorella sorokiniana : growth rate in rehation to light and temperature ( - graph adjusted to the model of Peeters \& Eilers 1978 ). 
Tableau 1. Modèle de croissance de Chlorella sorokiniana. Ajustement au modèle de Peeters \& Eilers (1978) : valeurs des paramètres.

Table 1. Growth model of Chiorella sorokiniana. Adjustment to the model of Peeters \& Eilers (1978) : values of parameters.

\begin{tabular}{lrrrrrr}
\hline Température $\left({ }^{\circ} \mathrm{C}\right)$ & 10 & 15 & 20 & 25 & 30 & 35 \\
$\mu \max \left(\mathrm{j}^{-1}\right)$ & 0,3 & 0,4 & 0,8 & 1,3 & 2,0 & 2,2 \\
Iopt $\left(\mu \mathrm{E} \cdot \mathrm{m}^{-2} \cdot \mathrm{s}^{-1}\right)$ & 30 & 75 & 150 & 230 & 300 & 320 \\
\multicolumn{1}{c}{$\beta$} & 1,5 & 2,5 & 2,3 & 2,1 & 1,6 & 1,3 \\
\hline
\end{tabular}

Tableau 2, Modèle de croissance de Chlorella sorokiniana. $\mu$ maxT $=f(T)$ et Iopt $T=f(T)$. Ajustement au modèle de Lehman et al. 1975 : valeurs des paramètres.

Table 2. Growth Model of Chlorella sorokiniana. $\mu$ maxT $=f(T)$ and lopt $T=f(T)$. Adjustment to the model of Lehman et al. 1975 : values of parameters.

\begin{tabular}{|c|c|c|c|}
\hline & \multicolumn{3}{|c|}{ Température $\left({ }^{\circ} \mathrm{C}\right)$} \\
\hline & $T^{\circ} \inf$ & $\mathrm{T}^{\circ} \mathrm{opt}$ & $T^{\circ}$ sup \\
\hline $\boldsymbol{\mu}^{\max _{\mathrm{T}}}=\mathbf{f}(\mathrm{T})$ & $\$ 1,7$ & 35 & 45 \\
\hline Iopt $_{T}=f(T)$ & 9,6 & 34,7 & 45 \\
\hline
\end{tabular}

Tableau 3. Principaux parametres spécifiques de croissance de Chlorella sorokiniana. Cas d'une photopériode de 15/9.

Table 3. Main specific parameters of growth of Chlorella sorokiniana. (15/9 light/dark photoperiod).

\begin{tabular}{ccccc}
$\mu^{\max }$ & $\mathrm{T}^{\circ}$ opt & lopt & $\mathrm{Q}_{10}$ & $\mathrm{TG}$ \\
$\left(\mathrm{j}^{-1}\right.$ & $\left({ }^{\circ} \mathrm{C}\right)$ & $\left(\mu \mathrm{E} \cdot \mathrm{m}^{-2} \cdot \mathrm{s}^{-1}\right)$ & & $(\mathrm{h})$ \\
2,2 & 35 & 320 & 2,5 & 7,5 \\
\hline
\end{tabular}

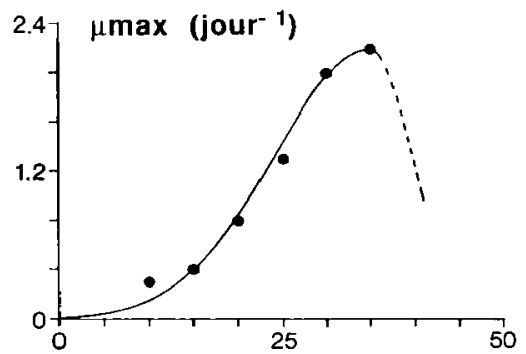

Température $\left({ }^{\circ} \mathrm{C}\right)$

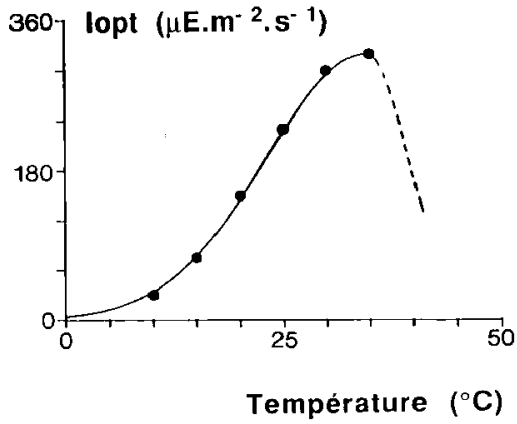

Fig. 3. Chlorella sorokiniana: Variation de l'intensité lumineuse optimale (lopt) en fonction de la temperature (ajusternent au modèle de Lehman et al. 1975).

Fig. 3. Chlorella sorokiniana : Variation of optimal light intensity (Iopt) in relation to temperature (graph adjusted to the model of Lehman et al. 1975). 


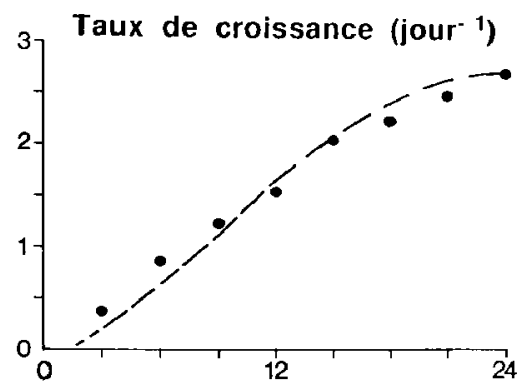

Heures d'éclairement

Fig. 4. Chlorella sorokiniana : Influence du nombre d'heures d'éclairement sur le taux de croissance (expérience réalisée à $30^{\circ} \mathrm{C}$ et à $\left.300 \mu \mathrm{E} \cdot \mathrm{m}^{-2} \cdot \mathrm{s}^{-1}\right)$.

Fig. 4. Chlorella sorokiniana: Influence of photoperiod on growth rate (experiment performed at $30^{\circ} \mathrm{C}$ and $300 \mu \mathrm{E} \cdot \mathrm{m}^{-2} \cdot \mathrm{s}^{-1}$ ).

L'ensemble de ces paramètres a été déterminé pour une photopériode de 15/9. Une expérience supplémentaire à $30^{\circ} \mathrm{C}$ et à $300 \mu \mathrm{E} \cdot \mathrm{m}^{-2} \cdot \mathrm{s}^{-1}$ a été réalisée pour replacer ces valeurs parmi une gamme de durée d'éclairement journaliers allant de 0 à 24 heures (Fig. 4). Les résultats montrent que le taux de croissance est d'autant plus important que le nombre d'heures d'éclairement est élevé ; la valeur maximale du taux de croissance mesuré est de $2,7 \mathrm{j}^{-1}$ pour un éclairement continu.

L'évolution du taux de croissance à $30^{\circ} \mathrm{C}$ en fonction du nombre d'heures d'éclairement peut être décrit par l'équation 5 :

$\mu 30, \mathrm{nH}=\mu(30,15 / 9)^{*}\left(\mathrm{~A}+1 /\left(\mathrm{B}+\mathrm{C}^{*} \mathrm{e}\left(\mathrm{D}-\mathrm{E}^{*} \mathrm{nH}\right)\right)\right.$

où $\mu 30, \mathrm{nH}$ représente le taux de croissance à $30^{\circ} \mathrm{C}$ pour un nombre $\mathrm{nH}$ d'heures d'éclairement. Ce résultat peut être extrapolé pour tous les cas de combinaisons Température-Photopériode $(T, n \mathbf{H})$ par la relation suivante :

$$
\begin{aligned}
& { }_{\mu} \max _{(\mathrm{T}, \mathrm{nH})}= \\
& \mu \max _{(T, 15 / 9)}\left(\mathrm{A}+1 /\left(\mathrm{B}+\mathrm{C}^{*} \mathrm{e}(\mathrm{D}-\mathrm{E} * \mathrm{nH})\right)(6)\right.
\end{aligned}
$$

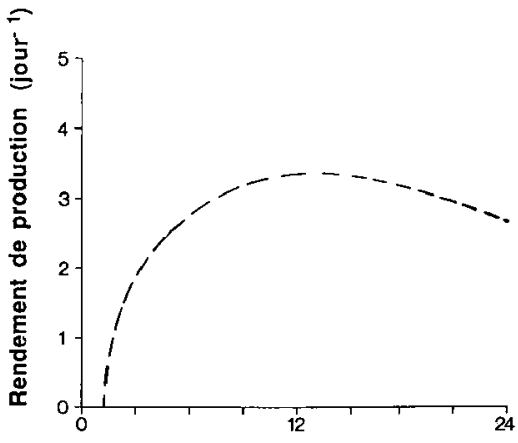

Nombre d'heures d'éclairement

Fig. 5, Chlorella sorokiniana : Rendement de production (taux de croissance pondéré, $\mu_{\mathrm{nH}}=\mu^{\mathrm{nH}} * 24 / \mathrm{nH}$ ) en fonction du nombre d'heures d'éclairement $(\mathrm{n} H)$, courbe théorique.

Fig. 5. Chlorello sorokiniana : Production yield in relation to the number of hours of light $(\mathrm{nH})$. Theoretical curve.

L'ajustement de ce modèle par les moindre carrés donne :

$\mathrm{A}=-0.35, \mathrm{~B}=0.548, \mathrm{C}=1.25, \mathrm{D}=0.693, \mathrm{E}=0.181$.

\section{Discussion}

Malgré le nombre important de travaux consacrés à la biologie et à la physiologie des espèces du genre Chlorella (Cf revue de Oh-Hama \& Miyachi 1988), les aspects écophysiologiques de certaines d'entre elles, notamment Chlorella sorokiniana restent très fragmentaires. L'étude qui a été réalisée représente en conséquence une source de données nouvelles permettant d'apprécier l'interdépendance entre la lumière et la température, ainsi que l'effet de la photopériode sur le taux de croissance de cette algue.

Les résultats obtenus pour une photopériode de $15 / 9$ montrent que cette espèce se caractérise par un taux de croissance maximal de $2,2 \mathbf{j}^{-1}$, correspondant à environ 3,1 divisions par jour. Cette valeur, enregistrée dans des conditions héliothermiques optimales $\left(35^{\circ} \mathrm{C}\right.$ et $\left.320{ }_{\mu} \mathrm{E} \cdot \mathrm{m}^{-2} \cdot \mathrm{s}^{-1}\right)$ est proche de celle reportée pour Chlorella pyrenoidosa Chick. $\left(2 \mathrm{j}^{-1}\right.$, Myers 1953, Hoogenhout \& Amesz 1965, Ogawa \& Aiba 
1981) et Chiorella ellipsoidea Gerneck. ( $2 \mathrm{j}^{-1}$, Tamiya et al. 1953), mais diffère très nettement de la valeur trouvée pour Chlorella vulgaris Beiger $\left(1 \mathrm{j}^{-1}\right)$ par Piquemal (1990). Selon Reynolds (1984), des taux de croissance aussi élevés $\left(\geqslant 2 \mathrm{j}^{-1}\right)$ laissent supposer que ces microalgues incorporent de manière relativement efficace le carbone photosynthétique dans le matériel cellulaire néoformé. Chez Chlorella sorokiniana, l'assimilation photosynthétique at teint à $35^{\circ} \mathrm{C}$ la valeur maximale de $6,7 \mathrm{mg}$ de carbone.mg de Chl. $\mathrm{a}^{-1} \cdot \mathrm{h}^{-1}$, soit environ $0,134 \mathrm{mg}$ de carbone.mg Carbone cellulaire-1.h-1 (Belkoura, résultats non publiés). Un tel taux d'assimilation devrait permettre théoriquement aux cellules de doubler leur biomasse en 7,5 heures, ce qui est en parfait accord avec les résultats obtenus ( $\mathrm{TG}=7,5$ heures, tableau 3).

L'évolution du taux de croissance avec l'intensité lumineuse et la température (Fig. 1 à 3) fait apparaître une interaction complexe entre ces trois paramètres. Parallèlement à la notion de température optimale de croissance - ce qui correspond pour Chlorella sorokiniana à $35^{\circ} \mathrm{C}$ - nos résultats mettent en évidence l'existence d'une intensité lumineuse optimale qui augmente régulièrement en fonction de la température, passant de 30 à $300 \mu \mathrm{E} \cdot \mathrm{m}^{-2} . \mathrm{s}^{-1}$ dans l'intervalle $10-35^{\circ} \mathrm{C}$. Ce type de réponse ne semble pas être spécifique aux chlorophycées, mais peut être observé également, à des degrés divers, chez les diatomées (Smayda 1969, Eppley 1977, Dauta 1982, Hartig \& Wallen 1986, Boumnich et al. 1990) et les cyanophycées (Foy et al. 1976, Krueger \& Eloff 1978, Post et al. 1985, Dauta et al. 1990).

L'ajustement quasi parfait des données expérimentales aux modèles mathématiques de Peeters \& Eilers (1978) et de Lehman et al. (1975) offre la possibilité de déterminer le taux de croissance de Chlorella sorokiniana à tout moment pour une combinaison d'éclairement (intensité, photopériode) et de température. L'étude de l'influence de la photopériode sur le taux de croissance montre que celui-ci est maximal sous un éclairement continu (Fig. 4). Cependant, les résultats obtenus en pondérant le taux de croissance enregistré sur 24 heures par la durée d'éclairement ( $\mu \mathrm{nH}$ corrigé $={ }_{\mu} \mathrm{nH}^{*} 24 / \mathrm{nH}$, Foy et al. 1976) montrent que, au-delà de 12 heures d'éclairement, le rendement de production baisse par rapport à la quantité d'énergie dispensée à la culture (Fig. 5). Ce type d'observation permet de montrer en particulier qu'au niveau des applications industrielles, le surcoût énergétique entraîné par un éclairement continu (par rapport par exemple à un éclairement $15 / 9$ ) ne correspond pas à un gain substantiel de production de biomasse.

La connaissance des caractéristiques écophysiologiques de Chlorella sorokiniana en culture constitue une première étape vers l'élaboration d'un modèle complet de croissance permettant de gérer la production de cette microalgue. Il est bien évident qu'une étude complémentaire portant sur l'analyse de la composition biochimique de cette espèce dans différentes situations expérimentales (effets de la température, de l'intensité lumineuse, de la photopériode, des carences éventuelles en azote et en phosphore, etc...) devrait permettre de préciser les conditions environnementales à privilégier pour orienter le métabolisme de cette souche vers la production du ou des métabolites souhaités.

\section{Remerciements}

Cette étude a été effectuée au laboratoire d'Hydrobiologie de Toulouse dans le cadre d'un stage de recherche financé par l'AUPELF.

\section{Travaux cités}

Becker E.W. 1986. - Nutritional properties of microalgae : potentials and contraints. In Richmond A. (Ed.), Handbook of microolgal Mass Cullure, CRC Press, Inc. Boca Raton, Florida, 349-419.

Borowitzka L. 1986. - Microalgae as sources of fine chemicals. Microbiological Sciences, $3(12): 372-375$.

Boumnich L.. Dauta A., Devaux J. \& Romagoux J.C. 1990. Influence de la lumière et de la température sur la croissance de quatre especes d'algues d'un lac eutrophe (Lac d'Aydat, Puy de Dôme, France). Annts Limnol., 26 (1) : 3-10.

Cohen Z. 1986. - Products from microalgae. In Richmond A. (Ed.), Handbook of microalgal Mass Culture, CRC Press, Inc. Boca Raton, Florida, 421-454.

Dauta A. 1982 . - Conditions de développenent du phytoplancton. Etude comparative du comportement de huit espèces en culture. 1. Détermination des paramètres de croissance en fonction de la lumière et de la température. Annis Limnol., 18 (3) : 217-262.

Dauta A., Devaux J., Piquemal F. \& Boumnich L. 1990. Growth rate of four freshwater algae in relation to light and temperature, Hydrobiologia, $207: 221-226$.

De Pauw N., Morales J. \& Persoone G. 1984. - Mass culture of microalgae in aquaculture systems : progress and constraints. Hydrobiologia, 116/117: 121-134.

Eppley R.W. 1977. - The growth and culture of diatoms. In Werner D. (Ed.), The Biology of diatoms, Botanical Monographs, $13: 24-64$.

Foy R.H., Gibson C.E. \& Smith R.V. 1976. - The influence of daylength, light intensity and temperature on the growth rates of planktonic blue-green algae. Br. Phycol. J., 11 : 151-163. 
Guerri M.M., Brunel L. \& Dauta A. 1981. - Interaction de la lumic̀re et de la température sur le taux de croissance de Scenedesmus crassus. Annls Limnol, 17 (2) : 97-104.

Hartig H. \& Wallen G. 1986. - The influence of light and temperature on growth and photosynthesis of Fragilaria crotonensis Kitton. Journal of Fresh water Ecology, 3 (3) : 371-382.

Hoogenhout H. \& Amesz J. 1965. - Growth rates of photosynthetic microorganisms in laboratory culture. Archiv. Microbiol., $50: 10-25$.

Krueger G.H.J. \& Eloff J.N. 1978. - The effect of temperature on specific growth rate and activation energy of Microcys$t$ is and Synechococcus isolates relevant to the onset of natural blooms. J. Limnol. Soc. Sth. Afr., 4 (1) : 9-20.

Lehman J.T., Botkin D.B. \& Likens G.E. 1975. - The assumptions and rationales of a computer model of phytoplankton population dynamics. Limnol. Oceanogr., $20: 343-364$.

MarkI H. 1980. - Modelling of algal production system. In G. Shelef \& C.J. Soeder (Eds) Algae Biomass, Elsevier Press, Amsterdam, 361-383.

Morgan K.C. \& Kalff J. 1979. - Effect of light and temperature interactions on growth of Cryptomonas erosa (Cryptophyceae). J. Phycol., $15: 127-134$.

Myers J. 1953. - Growth characteristics of algae in relation to the problems of mass culture. In J.S. Burlew (Ed.), Algal Culture from laboratory to pilot plant, , Washington D.C., Carnegie Institution, 37-54.

Ogawa T. \& Aiba S. 1981. - Bioenergetic analysis in mixotrophic growth growth in Chlorella vulgaris and Scenedesmus acutus. Biotechnology and Bioengineering., 23:1121-1132.

Oh-Hama T. \& Miyachi S. 1988. - Chlorella. In Borowitzka M.A. \& Borowitzka L.J. (Eds.), Microalgal biotechnology. Cambridge University Press, London, 3-26.
Peeters J.C. \& Eilers P. 1978. - The relationship between light intensity and photosynthes is : a simple rnathematical model. Hydrobiol. bull., 12 : 134-136.

Piquemal F, 1990. - Contribution à l'étude écophysiologique de quelques espèces d'algues en cullure. Thèse Doctorat Université Toulouse III, France.

Post A.F., De Witt R. \& Mur L.R. 1985. -- Interactions between temperature and light intensity on growth and photosynthesis on the cyanobacterium Oscillatoria agardhii. J. Planklon Res., 7 : $487+495$.

Reynolds C.S. - The ecology of freshwater phytoplankton. Cambridge University Press, London : 384 p.

Richmond A. 1986. - Microalgae of economic potentiel. In Richmond A. Ed., Handbook of microalgal mass cullure, CRC Press, Inc. Boca Raton, Florida, 199-243.

Smayda T.J. 1969. - Experimental observations on the influence of temperature, light and salinity on cell division of the marine diaton Detonula confervacea (Cleve). Gran., J. Phycol., 5 : 105-157.

Soeder C.J. 1980. Massive cultivation of microalgae : results and prospects. Hydrobiologia, $72: 197-209$.

Soong P. 1980. - Production and development of Chlorella and Spirulina in Taiwan. In Shelef G. \& Soeder C.J. (Eds.), Algae biomass, Elsevier Press, Amsterdam, 97-114.

Tamiya H., Hase E., Shibata K., Mituya A., Iwamura T., Nihei T. \& Sasa T. 1953, - Kinetics of growth of Chlorelia, with special reference to its dependence on quantity of available light and on temperature. In Burlew J.S. (Ed.), Algal culture from laboratory to pilot plant, Washington D.C., Carnegie Institution, 204-232. 